\title{
Efecto de un programa de ejercicios en síntomas músculo esqueléticos en docentes de primaria
}

\section{Effect of an exercise program on musculoskeletal symptoms in primary school teachers}

\author{
Rosmery V. Manrique-Collantes ${ }^{1, a}$ \\ ${ }^{1}$ Facultad de Medicina, Universidad Nacional Mayor de San Marcos. Lima, Perú. \\ ${ }^{a}$ Tecnólogo médico del área de terapia física y rehabilitación, ORCID: 0000-0002-9274-3930
}

An Fac med. 2020;81(4):391-7. / DOI: https://doi.org/10.15381/anales.v81i4.17761

\begin{abstract}
Correspondencia:
Rosmery Victoria Manrique Collantes manrique.collantes.@gmail.com

Recibido: 9 de agosto 2020

Aprobado: 29 de enero 2021

Publicación en línea: 15 de abril 2021

Conflictos de interés: La autora declara no tener conflictos de interés.
\end{abstract}

Fuente de financiamiento: Autofinanciado

Citar como: Manrique-Collantes $R$ Efecto de un programa de ejercicios en sintomas músculo esqueléticos en docentes de primaria. An Fac med. 2020;81(4):391-7. DOI: https://doi. org/10.15381/anales.v81i4.17761.

\section{Resumen}

Introducción. Los docentes de nivel primario están expuestos a riesgos físicos que pueden controlar mediante un programa de ejercicios, el cual busca promover mejoras para su salud de manera oportuna. Objetivos. Determinar el efecto de un programa de ejercicios en los síntomas músculo esqueléticos en docentes de nivel primario. Métodos. Se realizó un estudio experimental, donde se aplicó el cuestionario de Cornell. Resultados. Los docentes presentaron una mediana de 52 años de edad. Al inicio se encontró que los síntomas músculo esqueléticos más frecuentes de todos los docentes se encontraban en cuello $(50,8 \%)$, espalda baja $(41,5 \%)$, pie izquierdo $(35,4 \%$ y pie derecho $(33,9 \%)$; en la comparación final entre los grupos, se observó que la intensidad obtuvo diferencias significativas de valor de $p<0,01$ para cuello, hombro derecho/izquierdo, espalda alta, espalda baja, rodilla derecha/izquierda. Cabe destacar que la diferencia de datos iniciales y finales, el grupo intervención obtuvo $25 \%$ a más para las zonas de cuello, espalda baja, hombro derecho/izquierdo y pie derecho; mientras que en el grupo control, el 18,2\% empeorarían en la zona de la espalda baja. Conclusiones. El programa de ejercicios disminuyó significativamente los sintomas musculo esqueléticos de los docentes del grupo intervención, mientras que en el grupo control se mantuvieron similares o aumentaron.

Palabras clave: Ejercicio Físico; Salud Laboral; Personal Docente; Dolor Musculoesquelético (fuente: DeCS BIREME).

\section{Abstract}

Introduction. Primary-level teachers are exposed to physical risks that they can control through an exercise program, which seeks improved promotions for their health in a timely manner. Objectives. To determine the effect of an exercise program on the musculoskeletal symptoms in primary school teachers. Methods. An experimental study was carried out, where the Cornell questionnaire was applied. Results. The teachers had a median age of 52 years. Initially, it was found that the most frequent musculoskeletal symptoms of all teachers were found in the neck $(50,8 \%)$, lower back $(41,5 \%)$, left foot $(35,4 \%)$ and right foot $(33,9 \%)$; in the final comparison between the groups, it is observed that the intensity obtained significant differences of $p$ value $<0,01$ for neck, right/left shoulder, upper back, lower back, right/left knee. It is worth noting in the difference in initial and final data, the intervention group obtained $25 \%$ or more for the neck, lower back, right/ left shoulder and right foot areas; while in the control group, 18,2\% would worsen in the lower back area. Conclusions. The exercise program decreased significantly the musculoskeletal symptoms of the teachers in the intervention group, while in the control group were similar or increased.

Keywords: Exercise; Occupational Health; Educational Personnel; Musculoskeletal Pain (source: MeSH NLM). 


\section{INTRODUCCIÓN}

Los trastornos músculo esqueléticos son la principal causa de discapacidad mundial; así, una de cada tres o cinco personas, sufre una afección osteoarticular o muscular dolorosa y discapacitante, además de afectar la vida social de las personas ${ }^{(1)}$. Esto se produce cuando el esfuerzo mecánico es superior a la capacidad de resistencia de los componentes del aparato locomotor, dado que después de hacer un esfuerzo físico es normal que se experimente cierta fatiga. Los síntomas aparecen como molestias propias de la vida (2); aun así, la intensidad y la duración del trabajo pueden guardar relación con posibles alteraciones en el sistema osteomuscular, pudiéndose convertir en enfermedades ocupacionales, ya que son de inicio lento y persistente, como resultado de repetidas exposiciones laborales ${ }^{(3)}$.

El Ministerio de Salud del Perú bajo la Resolución Ministerial N480-2008, tiene aprobado un listado de enfermedades ocupacionales dentro de las cuales se encuentran las enfermedades provocadas por posturas forzadas y movimientos repetitivos ${ }^{(4)}$; incluso el Ministerio de Educación del Perú en la Encuesta Nacional de Docentes del 2018, reflejó altos porcentajes en las exigencias físicas, sea para estar de pie la mayor parte del tiempo o mantener una postura incómoda durante su tiempo de trabajo ${ }^{(5)}$. A esto se agrega que los síntomas músculo esqueléticos (SME) en los docentes han sido evaluados más en los últimos años, buscando asociación a factores demográficos, laborales y de riesgo psicosocial ${ }^{(6,7,8)}$, pero siempre tienen como base que son de origen multifactorial; respecto a las zonas corporales afectadas, se encontró un estudio en Brasil (9), que los docentes presentaron mayores SME en el tronco y miembros superiores, afectando las actividades diarias y dificultando las actividades de trabajo. Otro estudio en Perú ${ }^{(10)}$, reportó que las zonas más prevalentes fueron lumbar, cervical y tobillo pie.

Según el Instituto Nacional de Estadística e Informática existen aproximadamente 210 mil docentes en nivel primario ${ }^{(11)}$. En el año 2011, la Organización de las Naciones Unidas para la Edu- cación, Ciencia y Cultura manifestó que el cuerpo docente no crecía en la misma proporción que el número de alumnos matriculados ${ }^{(12)}$, y aún 5 años más tarde, se encuentra que existe una provisión de docentes inadecuada ${ }^{(13)}$, con lo que en la actualidad se tiene hasta 35 niños por aula en los colegios públicos, que aumenta la demanda de atención y el esfuerzo físico del docente, y que podría repercutir sobre la calidad de educación (12,13).

Existen estudios que promueven la utilización de medidas de promoción y prevención, como es el caso de los programas de ejercicios para contrarrestar las altas prevalencias de SME (14-17), y para mejorar la capacidad laboral ${ }^{(18,19)}$; sin embargo, se debe tener en cuenta que esta medida debe ser específica en función del ritmo y exigencia de cada trabajo ${ }^{(20)}$. Si bien existen folletos informativos para prevenir enfermedades ocupacionales en docentes, establecidos por el Ministerio de Educación del Perú (21), no existe evidencia del efecto en SME por alguna actividad de prevención en las instituciones educativas de nivel primario en Lima, siendo una oportunidad desarrollar este estudio en uno de sus distritos que es Surquillo.

La implementación del programa de ejercicios es una medida para fomentar una cultura de prevención ${ }^{(22)}$, dado que todos los lugares de trabajo deben de cuidar la salud de sus colaboradores tal como lo establecen 2 leyes peruanas, Ley 29783, Ley de Seguridad y Salud en el trabajo ${ }^{(23)}$ y la Ley 28044, Ley de Educación (24); en su artículo 49 y 56 respectivamente.

Por ello, se realizó la elaboración de un programa de ejercicios supervisados con el objetivo de determinar el efecto de su implementación en el grupo intervención frente al grupo control en la prevalencia de ssíntomas músculo esqueléticos en docentes de nivel primario en un distrito de Lima.

\section{MÉTODOS}

\section{Diseño de la investigación}

Se realizó un estudio experimental con grupo control, aleatorizado, con medición inicial y final ${ }^{(25)}$.

\section{Población y muestra}

Se incluyeron 7 instituciones educativas públicas de nivel primario del distrito de Surquillo, Lima, Perú, donde laboraban un total de 90 docentes. Para la selección de muestra se consideró a las personas que cumplieran con los siguientes criterios: acepten participar de las intervenciones, tener un mínimo de 5 años de experiencia laboral, 30 horas mínimas de trabajo a la semana, hasta los 65 años de edad, y que no se encuentren embarazadas; excluyendo a quienes tengan alguna secuela músculo esquelética o lesión aguda, se encuentren con descanso médico, o tomen con regularidad medicamentos analgésicos. Así, participaron del estudio 70 docentes.

Se establecieron códigos a cada participante, se colocó en orden según años de experiencia laboral y finalmente se seleccionaron por medio de una tabla de números aleatorios; designando la mitad de participantes al grupo intervención y a la otra mitad al grupo control.

\section{Procedimientos y mediciones}

El estudio se realizó entre los meses de abril a agosto del 2019. Se implementó un programa de ejercicios con una duración de 8 semanas, considerando lo reportado en artículos previos ${ }^{(14-17)}$, con una frecuencia de 2 veces a la semana ${ }^{(26,27)}$, y que se realizaron durante los turnos de recreo o educación física, antes del dictado de clase o al finalizar esta.

El programa estuvo conformado por ejercicios de respiración, estiramiento muscular y fortalecimiento progresivo, con una duración de 10 minutos; además, 2 sesiones de estiramiento pasivo de miembros inferiores de 20 minutos. Los ejercicios respiratorios se realizaron al inicio y final de cada día con frecuencia de 5 repeticiones; los estiramientos musculares, 3 repeticiones de 10 a 15 segundos; el fortalecimiento, 3 series de 10 repeticiones cada uno; el estiramiento pasivo de miembros inferiores se realizó 3 a 5 veces de 15 a 20 segundos. Fue orientado y supervisado por un fisioterapeuta.

Al grupo intervención se le brindó el programa de ejercicios y al grupo control no se le brindó ningún programa. 
Las características del SME fueron medidas una semana antes y una semana después de la intervención a todos los participantes (ambos grupos). Todos los docentes tuvieron similares factores de riesgo como mobiliario, temperatura, ruido, iluminación, carga de trabajo físico y mental.

Se utilizó el Cuestionario de Malestares Musculo Esqueléticos de Cornell para trabajadores de pie, versión en español, que tiene un coeficiente de alfa de Cronbach de 0,8; un coeficiente de correlación de Spearman 0,4 a 0,8; y un coeficiente de concordancia de Kappa de 0,6 a 0,9 ${ }^{(28)}$. El análisis de datos se realizó según un estudio previo ${ }^{(29)}$. Dicho instrumento evalúa 20 partes del cuerpo, con las siguientes características del síntoma musculo esquelético para cada una: frecuencia (nunca, 1-2 veces la semana pasada, 3-4 veces la semana pasada, una vez cada día, o varias veces al día), intensidad (ligeramente incómodo, moderadamente incómodo o muy incómodo), e interferencia de la capacidad laboral (casi nada, interfirió ligeramente, interfirió contundentemente) que para el cuestionario se le denomina productividad. El puntaje total se halló de la multiplicación de estas 3 características. Se consideraron otras variables como el sexo, los años de experiencia laboral y la edad.

\section{Análisis estadístico}

Los resultados se ingresaron a una base de datos en Excel; luego del control fueron exportados al programa SPSS v22. Debido a que existen datos jerarquizados en las características del SME, el tamaño de muestra es pequeña para cada grupo y la variable síntomas músculo esqueléticos tiene valores cualitativos, se utilizó la prueba de kolmogorov- Smirnov para comprobar la normalidad de todos los datos, mostrando que la muestra no tuvo una distribución normal en sexo, años de experiencia laboral, en las partes del cuerpo según presencia, frecuencia, intensidad y productividad, salvo en la edad. Se determinó que la estadística a aplicar serian pruebas no paramétricas (McNemar, $U$ de Man Whitney y Wilcoxon).

Para mostrar una mejor presentación de los datos, la variable nominal (presencia) se expresó en frecuencias y porcentajes. Se describió el puntaje inicial y se demostró su homogeneidad con la prueba de Chi cuadrado. Se comparó la diferencia entre los datos iniciales y finales de cada grupo con la prueba de McNemar. Asimismo, las variables ordinales (frecuencia, intensidad, productividad) se expresaron de forma independiente con la media. Para la comparación de datos finales en cada grupo se aplicó la prueba de U de Man Whitney. Mientras que la prueba de Wilcoxon se utilizó para el valor del puntaje total obtenido de las variables ordinales ya mencionadas, comparando los datos iniciales y finales independientemente de cada grupo.

\section{Aspectos éticos.}

El estudio fue aprobado por el Comité de Ética en Investigación de la Universidad Nacional Mayor de San Marcos (CEl/19-0069) y por la Dirección de cada institución educativa que participó en el estudio. Los participantes fueron in- formados de los objetivos del estudio y firmaron el consentimiento informado, acorde con los preceptos de la Declaración de Helsinki, la cual garantiza ningún riesgo, confidencialidad y la participación de forma voluntaria. Luego de finalizar el estudio, el grupo control que no fue intervenido, también realizó el programa de ejercicios.

\section{RESULTADOS}

Participaron del estudio 35 docentes en el grupo de intervención y 35 en el grupo control. Durante la intervención 5 personas abandonaron el estudio, siendo al final 32 docentes el grupo intervención y 33 docentes el grupo control (Figura1).

La mediana de la edad fue de 52 años, y 28 los años de experiencia, siendo el sexo femenino el más frecuente con 92,3\%.

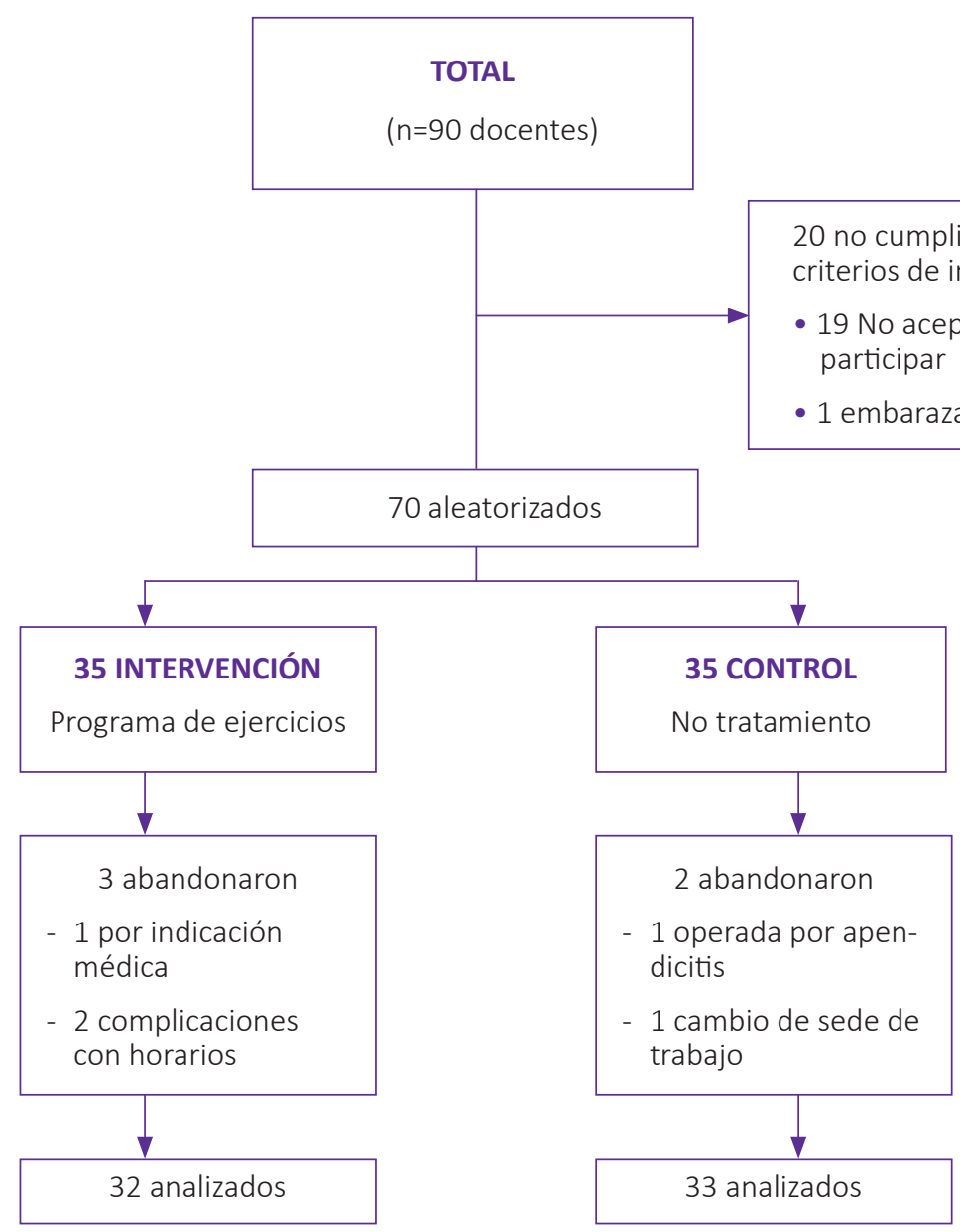

Figura 1. Diagrama de flujo de docentes que participaron del estudio, según grupo de intervención y control, sobre el efecto de un programa de ejercicios en los síntomas músculo esqueléticos. 
En la tabla 1 se muestra los datos iniciales en la presencia de SME de cada grupo con respecto a las zonas corporales, encontrándose para el total de los datos que, las zonas más predominantes fueron cuello $(50,8 \%)$, espalda baja $(41,5 \%)$, se- guidas de pie izquierdo $(35,4 \%)$ y derecho $(33,9 \%)$; todas las zonas fueron evaluadas comprobando su homogeneidad entre grupos con valor de $p>0,05$.

En la tabla 2, se muestra la diferencia de los valores iniciales y finales en la pre-

Tabla 1. Presencia inicial de SME, según zona corporal, en los grupos de docentes de instituciones educativas públicas de nivel primario de Surquillo, Lima, 2019.

\begin{tabular}{|c|c|c|c|c|c|c|}
\hline & & \multirow{3}{*}{$\begin{array}{l}\text { Total } \\
n=65\end{array}$} & \multirow{3}{*}{$\%$} & \multicolumn{2}{|c|}{ Inicial } & \multirow{3}{*}{ Valor $\mathrm{p}^{*}$} \\
\hline & & & & $\begin{array}{c}\text { Grupo } \\
\text { intervención }\end{array}$ & $\begin{array}{l}\text { Grupo } \\
\text { control }\end{array}$ & \\
\hline & & & & $n=32$ & $n=33$ & \\
\hline Cuello & & 33 & 50,8 & 17 & 16 & 0,900 \\
\hline \multirow[t]{2}{*}{ Hombro } & $\mathrm{D}$ & 18 & 27,7 & 9 & 9 & 1,000 \\
\hline & 1 & 16 & 24,6 & 10 & 6 & 0,350 \\
\hline Espalda alta & & 20 & 30,8 & 8 & 12 & 0,469 \\
\hline Espalda baja & & 27 & 41,5 & 16 & 11 & 0,266 \\
\hline Caderas & & 12 & 18,5 & 6 & 6 & 1,000 \\
\hline \multirow[t]{2}{*}{ Rodilla } & D & 19 & 29,2 & 8 & 11 & 0,641 \\
\hline & I & 17 & 26,2 & 9 & 8 & 0,941 \\
\hline \multirow[t]{2}{*}{ Pantorrilla } & $\mathrm{D}$ & 14 & 21,5 & 9 & 5 & 0,332 \\
\hline & 1 & 14 & 21,5 & 9 & 5 & 0,332 \\
\hline \multirow[t]{2}{*}{ Pie } & $D$ & 22 & 33,9 & 10 & 12 & 0,862 \\
\hline & I & 23 & 35,4 & 12 & 11 & 0,927 \\
\hline
\end{tabular}

D: derecho, I: izquierdo

* Prueba de Chi cuadrado

Tabla 2. Diferencia de valores iniciales y finales de la presencia de SME, por grupos, según zona corporal, en docentes de instituciones educativas públicas de nivel primario de Surquillo, Lima, 2019.

\begin{tabular}{|c|c|c|c|c|c|c|c|}
\hline & \multicolumn{4}{|c|}{ Grupo intervención } & \multicolumn{3}{|c|}{ Grupo control } \\
\hline & & Inicial - Final & $\%(\mathrm{GI})$ & Valor $\mathrm{p}^{*}$ & $\begin{array}{c}\text { Inicial - } \\
\text { Final }\end{array}$ & $\%(G C)$ & Valor $\mathrm{p}^{*}$ \\
\hline Cuello & & 10 & 31,3 & 0,002 & -4 & 12,1 & 0,219 \\
\hline \multirow[t]{2}{*}{ Hombro } & $\mathrm{D}$ & 8 & 25,0 & 0,008 & -4 & 12,1 & 0,219 \\
\hline & 1 & 8 & 25,0 & 0,008 & -3 & 9,1 & 0,250 \\
\hline Espalda alta & & 6 & 18,8 & 0,031 & -5 & 15,2 & 0,063 \\
\hline Espalda baja & & 11 & 34,4 & 0,001 & -6 & 18,2 & 0,031 \\
\hline Caderas & & 3 & 9,4 & 0,250 & -3 & 9,1 & 0,375 \\
\hline \multirow[t]{2}{*}{ Rodilla } & D & 6 & 18,8 & 0,031 & -2 & 6,1 & 0,500 \\
\hline & 1 & 7 & 21,9 & 0,016 & -2 & 6,1 & 0,500 \\
\hline \multirow[t]{2}{*}{ Pantorrilla } & D & 7 & 21,9 & 0,016 & 1 & 3,0 & 1,000 \\
\hline & । & 6 & 18,8 & 0,031 & 0 & 0,0 & 1,000 \\
\hline \multirow[t]{2}{*}{ Pie } & $\mathrm{D}$ & 8 & 25,0 & 0,008 & 3 & 9.1 & 0,375 \\
\hline & I & 7 & 21,9 & 0,016 & 3 & 9.1 & 0,375 \\
\hline
\end{tabular}

GI: grupo intervención, GC: grupo control, D: derecho, I: izquierdo

* Prueba de Mc Nemar

sencia de SME para cada grupo, encontrándose que el grupo intervención tuvo reducciones en todos los segmentos. Solo un 9,4\% en caderas no alcanzó ser significativo según la prueba estadística aplicada obteniendo un $p=0,250$. En el grupo intervenido un $25 \%$ a más en cuello, espalda baja, hombro derecho/ izquierdo y pie derecho; mientras que en el grupo control, la zona que más empeoró fue la espalda baja con un cambio de $18,2 \%(p=0,031)$.

En la tabla 3 se muestra la comparación de los datos finales de la frecuencia, intensidad y productividad de los SME en las zonas corporales entre los grupos. Se observó similitud en la zona de pantorrillas derecha e izquierda con un valor de $p>0,05$. Específicamente se determinó que el factor de la intensidad tuvo diferencias significativas de valor de $p<0,01$ para cuello, hombro derecho/izquierdo, espalda alta, espalda baja, rodilla derecha/izquierda.

En la tabla 4 se detalla el efecto del programa de ejercicios en el SME mediante la comparación del puntaje total inicial y final en cada grupo según zona corporal, encontrándose que el grupo intervención mostró disminuciones en el dato final de todas las zonas corporales y estos cambios fueron significativos con un $p<0,05$; mientras que el grupo control tuvo datos similares $(p>0,05)$ en su mayoría.

\section{DISCUSIÓN}

El estudio mostró que los datos iniciales de los grupos fueron similares, luego se compararon las características del síntoma músculo esquelético según la frecuencia, intensidad y productividad (interferencia en la capacidad laboral) entre el grupo intervención y control, cuyos resultados contribuyen en cumplir con el objetivo del estudio.

Respecto a la presencia inicial de SME del total de participantes, se obtuvo que las zonas más predominantes fueron cuello, espalda baja, pie derecho/izquierdo; esto concuerda con un estudio realizado en docentes de nivel primario y 
Tabla 3. Datos finales en la frecuencia, intensidad y productividad del SME, según zonas corporales, entre los grupos de docentes de instituciones educativas públicas de nivel primario de Surquillo, Lima, 2019.

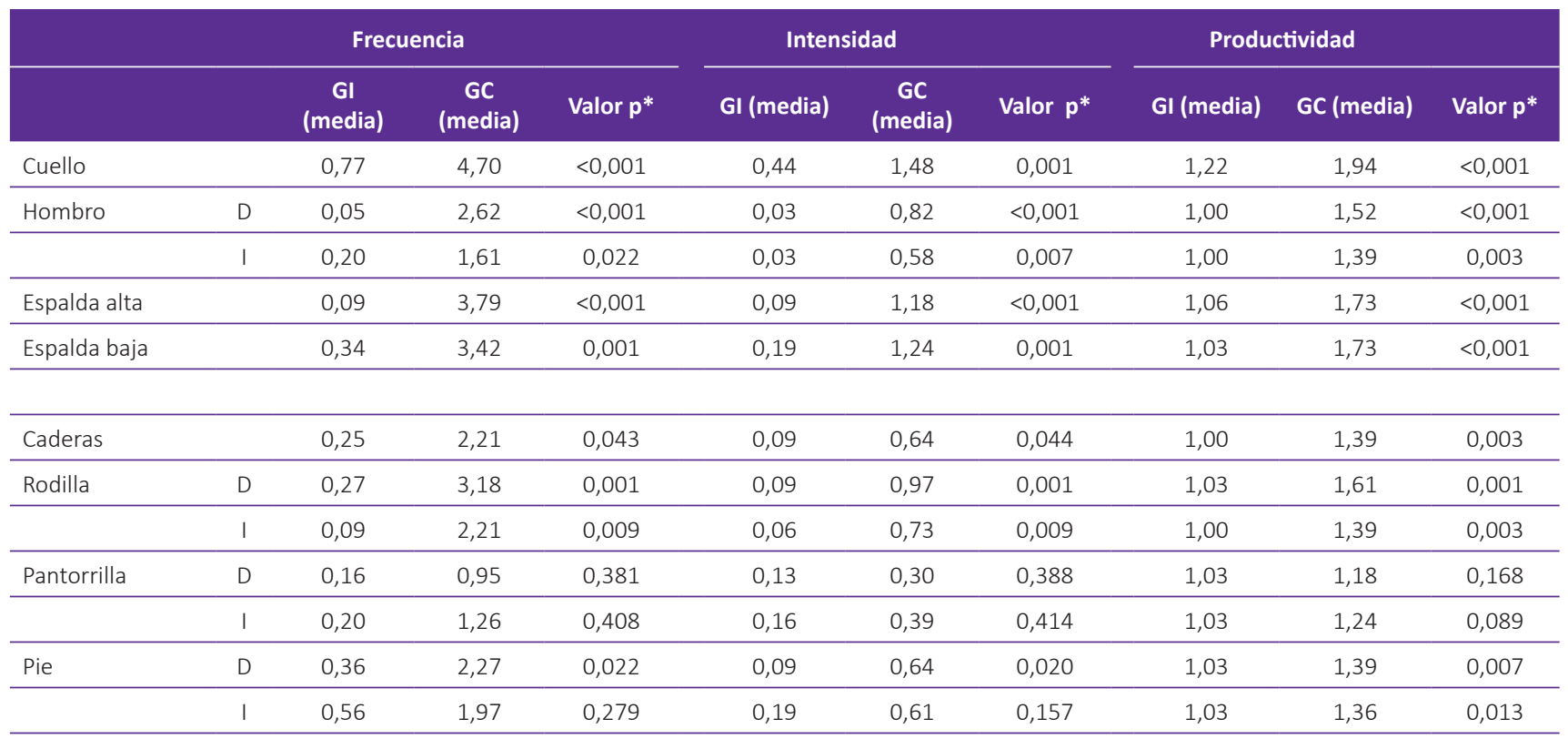

GI: grupo intervención, GC: grupo control, D: derecho, I: izquierdo

*Prueba U de Man Whitney

secundario en otro distrito de lima ${ }^{(10)}$, a los docentes como: la postura mantambién concuerdan con las dos primeras zonas para una población de enfermeras ${ }^{(30)}$, quienes tuvieron similares características ergonómicas de trabajo tenida, posturas incómodas, demanda de esfuerzo físico por la cantidad de personas que atienden, cabeza usualmente dirigida hacia abajo.
En relación al efecto del programa de ejercicio en docentes del grupo intervención, se obtuvo reducciones de $25 \%$ a más sobre la presencia de SME para las zonas de cuello, espalda baja, hombro

Tabla 4. Efecto del programa de ejercicios en el SME mediante la comparación del puntaje total, inicial y final, en cada grupo, según zona corporal en los docentes de instituciones educativas públicas de nivel primario de Surquillo, Lima, 2019.

\begin{tabular}{|c|c|c|c|c|c|c|c|}
\hline & & \multicolumn{2}{|c|}{ Grupo intervención } & \multirow{3}{*}{ Valor $p^{*}$} & \multicolumn{2}{|c|}{ Grupo control } & \multirow{3}{*}{ Valor $p^{*}$} \\
\hline & & Inicial & Final & & Inicial & Final & \\
\hline & & (media) & (media) & & (media) & (media) & \\
\hline Cuello & & 18,78 & 4,64 & 0,002 & 21,94 & 31,47 & 0,021 \\
\hline \multirow[t]{2}{*}{ Hombro } & $\mathrm{D}$ & 10,34 & 0,05 & 0,008 & 15,18 & 17,77 & 0,059 \\
\hline & 1 & 11,09 & 0,05 & 0,005 & 10,27 & 12,71 & 0,043 \\
\hline Espalda alta & & 9,38 & 0,05 & 0,012 & 13,79 & 24,33 & 0,009 \\
\hline Espalda baja & & 15,36 & 0,44 & $<0,001$ & 10,73 & 23,58 & 0,004 \\
\hline Caderas & & 8,84 & 0,25 & 0,028 & 13,94 & 16,20 & 0,596 \\
\hline \multirow[t]{2}{*}{ Rodilla } & $\mathrm{D}$ & 11,31 & 0,59 & 0,012 & 25,15 & 23,41 & 0,893 \\
\hline & 1 & 8,78 & 0,09 & 0,008 & 18,48 & 16,76 & 0,713 \\
\hline \multirow[t]{2}{*}{ Pantorrilla } & $\mathrm{D}$ & 9,34 & 0,53 & 0,008 & 8,00 & 7,36 & 0,317 \\
\hline & 1 & 9,66 & 0,58 & 0,008 & 8,00 & 10,09 & 0,655 \\
\hline \multirow[t]{2}{*}{ Pie } & $\mathrm{D}$ & 9,53 & 0,50 & 0,005 & 18,82 & 15,06 & 0,172 \\
\hline & । & 14,25 & 0,77 & 0,002 & 17,61 & 14,45 & 0,223 \\
\hline
\end{tabular}

D: derecho, I: izquierdo

*Prueba de Wilcoxon 
derecho/izquierdo. Estos resultados se asemejan con lo encontrado en estudios en personal de oficina ${ }^{(15,17)}$; a pesar de ser una población con actividades distintas a los docentes, cabe resaltar que su intervención también fue supervisada por fisioterapeutas, quienes se encargaron de velar que los ejercicios sean específicos a su labor. Respecto al grupo control, se encontró que la zona de la espalda baja fue la más afectada de no recibir la intervención del programa de ejercicios, esto concuerda con el estudio realizado en el personal de enfermería ${ }^{(30)}$, quienes, pese a realizar cambios ergonómicos en su lugar de trabajo, no priorizó a los ejercicios físicos, por lo que sugiere dar énfasis a estos.

Los datos finales de las características del SME fueron similares para las zonas de pantorrilla derecha/izquierda; esto podría deberse a factores no controlados, como por ejemplo el calzado, pues según un estudio en trabajadores que se encontraban usualmente de pie, un calzado inestable tendría intervención positiva en la disminución de incomodidad de dicha zona ${ }^{(31)}$.

Centrándonos en el factor específico de la intensidad final del SME, tuvo mayores diferencias significativas para las zonas de cuello, hombro derecho/izquierdo, espalda alta y espalda baja; dichos datos concuerdan con Cáceres-Muñoz y col. ${ }^{(17)}$ y Freitas-Swerts y col. ${ }^{(15)}$, a pesar de que sus intervenciones fueron de mayor tiempo, sea en las veces por semana o en la cantidad de semanas respectivamente, lo que respalda el argumento que el aumento de ejercicios disminuye la intensidad de dolor (32). Pero no solo se busca evidenciar cambios sino que estos lleguen a implementarse durante el tiempo de trabajo.

Cabe resaltar que el efecto del programa de ejercicios se reflejó significativamente en la disminución que existió entre los datos iniciales y finales del puntaje total de SME en el grupo intervención; esto concuerda con el estudio de Shariat y col. ${ }^{(16)}$ donde demostró que la intervención de solo estiramientos tiene mejores resultados que combinarlos con modificaciones ergonómicas, o no intervenir.

Una limitación del estudio fue que los participantes pertenecían a 7 lugares di- ferentes, esto podría afectar la similitud de sus datos, pero todos se desenvolvían en instituciones educativas públicas de nivel primario, de un mismo distrito, con las mismas condiciones laborales, y sus datos iniciales resultaron ser homogéneos; otra, es la disposición de los docentes. Además, la falta de cegamiento de análisis de datos.

Se concluye que el programa de ejercicios disminuyó significativamente los síntomas músculo esqueléticos de los docentes del grupo intervención, mientras que en el grupo control se mantuvieron similares o aumentaron. Recomendamos realizar más investigaciones de implementación de programas de ejercicios en docentes para reducir los síntomas musculo esqueléticos, con muestras representativas, a fin de obtener resultados inferenciales.

\section{AGRADECIMIENTOS}

A los profesores, directoras y personal administrativo de las instituciones educativas públicas de Surquillo $N^{\circ} 7031,7028$, 7027, 7024, 7022, 7014 y 7012 por su colaboración y cooperación para hacer realidad el estudio.

\section{REFERENCIAS BIBLIOGRÁFICAS}

1. The Burden of Musculoskeletal Diseases in the United States: Prevalence, Societal and Economic Cost [Internet]. United States: United States Bone and Joint Initiative; 2016 [citado 4 febrero 2020]. Disponible en: https://www.boneandjointburden. org/docs/BMUSExecutiveSummary2016.pdf

2. Prevención de trastornos musculoesqueléticos en el lugar de trabajo [Internet]. Francia: Organización Mundial de la Salud; 2004 [citado 23 febrero 2020]. Disponible en: http://www.who.int/occupational_health/publications/en/pwh5sp.pdf?ua=1

3. Manual de salud ocupacional. Dirección ejecutiva de Salud ocupacional [Internet]. Lima: Dirección General de Salud Ambiental, Dirección Ejecutiva de Salud Ocupacional; 2005 [citado 20 marzo 2020]. Disponible en: http://www.digesa.minsa. gob.pe/publicaciones/descargas/manual_deso. PDF

4. Resolución Ministerial № 480-2010/MINSA. Norma Técnica de Salud que establece el Listado de Enfermedades Profesionales. El Peruano (14-7-2008). [citado 3 febrero 2020]. Disponible en: http://www. gacetajuridica.com.pe/servicios/normaspdf_2008/ julio/17-07-08/17-07-2008.pdf

5. ESCALE: Unidad de estadistica educativa. Encuesta Nacional a Docentes de Instituciones Educativas Públicas y Privadas 2018. [dataset]. 29 abril 2019 [citado 2 marzo 2020]. Ministerio de
Educación del Perú. Disponible en: http://escale. minedu.gob.pe/uee/-/document_library_display/ GMv7/view/4385260

6. Ceballos A, Carvalho F. Verbal Aggression Against Teacher and Upper Extremity Musculoskeletal Pain. Saf Health Work. 2020;11(2): 187-192. DOI: 10.1016/j.shaw.2020.02.003

7. Ehsani F, Mohseni-Bandpei M, Fernández-De-Las-Peñas C, Javanshir K. Neck pain in Iranian school teachers: Prevalence and risk factors. J Bodyw Mov Ther. 2018; 22(1):64-68. DOI: 10.1016/j.jbmt.2017.04.003

8. Constantino $D$, Júnior $R$, Ribeiro $E$, de Oliveira A. Factors associated with musculoskeletal disorders and disability in elementary teachers: $\mathrm{A}$ cross-sectional Study. J Bodyw Mov Ther. 2019; 23(3):658-665. DOI: 10.1016/j.jbmt.2018.05.009

9. Calixto M, Azevedo P, Da Silva D, Tavares P. Prevalência de sintomas osteomusculares e suas relações com o desempenho ocupacional entre professores do ensino médio público. Cadernos Brasileiros de Terapia Ocupacional. 2015;23(3): 533-542. DOI: 10.4322/0104-4931.ctoAO0551

10. Manrique R. Influencia de la postura bípeda sostenida en sintomas musculoesqueléticos en profesores del colegio "Antonia Moreno de Cáceres" de San Juan de Lurigancho 2017 [Tesis de Licenciatura]. Lima, Perú: Universidad Nacional Mayor de San Marcos, 2018. Disponible en: http://cybertesis.unmsm.edu.pe/bitstream/handle/ cybertesis/7938/Manrique_cr\%20-\%20Resumen. pdf? sequence $=1$ \&isAllowed $=y$

11. Instituto Nacional de Estadística e Informática. Docentes en el sistema educativo, según nivel y modalidad, 2008-2018. [dataset] 30 octubre 2019 [citado 21 abril 2020]. Presidencia del consejo de ministros. Disponible en: https://www.inei.gob.pe/ estadisticas/indice-tematico/education/

12. La UNESCO y la educación: Toda persona tiene derecho a la educación [Internet]. Francia: Organización de las Naciones Unidas para la Educación, Ciencia y Cultura; 2011. [citado 22 abril 2020]. Disponible en: http://unesdoc.unesco.org/ images/0021/002127/212715s.pdf

13. La educación al servicio de los pueblos y el planeta [Internet]. Francia: Organización de las Naciones Unidas para la Educación, Ciencia y Cultura; 2016. [citado 23 marzo 2020]. Disponible en: https:// unesdoc.unesco.org/ark:/48223/pf0000248526

14. Szeto G, Wong T, Law R, Lee E, Lau T, So B, et al. The impact of a multifaceted ergonomic intervention program on promoting occupational health in community nurses. Appl Ergon. 2013;44(3):41422. DOI: 10.1016/j.apergo.2012.10.004

15. Freitas-Swerts F, Robazzi M. The effects of compensatory workplace exercises to reduce work-related stress and musculoskeletal pain. Rev Lat Am Enfermagem. 2014;22(4):629-636. DOI: 10.1590/0104-1169.3222.2461

16. Shariat A, Cleland J, Danaee M, Kargarfard M, Sangelaji B, Mohd S. Effects of stretching exercise training and ergonomic modifications on musculoskeletal discomforts of office workers: a randomized controlled trial. Braz J Phys Ther. 2017;22(2):144153. DOI: 10.1016/j.bjpt.2017.09.003

17. Cáceres-Muñoz V, Magallanes-Meneses A, TorresCoronel D, Copara-Moreno P, Escobar-Galindo M, Mayta-Tristán P. Efecto de un programa de pausa activa más folletos informativos en la disminución de molestias musculoesqueléticas en trabajadores administrativos. Rev Peru Med Exp 
Salud Publica. 2017;34(4):611-8. DOI: 10.17843/ rpmesp.2017.344.2848

18. Oliv S, Noor A, Gustafsson E, Hagberg M. A lower level of physically Demanding Work is associated with excellent work ability in men and women with neck pain in different age groups. Saf Health Work. 2017;8(4):356-363. DOI: 10.1016/j. shaw.2017.03.004

19. Ramalho-Pires de Almeida MÁ, Ábalos-Medina GM, Villaverde-Gutiérrez C, Gomes-de Lucena NM, Ferreira-Tomaz A, Perez-Marmol JM. Effects of an ergonomic program on the quality of life and work performance of university staff with physical disabilities: A clinical trial with three-month follow-up. Disabil Health J. 2019;12(1):58-64. DOI: 10.1016/j. dhjo.2018.07.002

20. Nogareda S, Bestratén M. El descanso en el trabajo (I): Pausas [Internet]. Madrid: Instituto Nacional de Seguridad e Higiene en el Trabajo. 2011 [citado 23 enero 2020]. Recuperado de: https://www.insst. es/documents/94886/328579/916w.pdf/f558c8641df9-4e42-ad11-7db78b6a7a35

21. Prevención de enfermedades: Pausas Activas [Internet]. Lima: Ministerio de Educación del Perú. 2018. [citado 22 febrero 2020]. Disponible en: http://www.minedu.gob.pe/politicas/docencia/ salud-prevencion-enfermedades.php
22. Rodríguez C. Los convenios de la OIT sobre seguridad y salud en el trabajo: Una oportunidad para mejorar las condiciones y el medioambiente de trabajo [Internet] Buenos Aires: Centro Internacional de Formación de la Organización Internacional del Trabajo. 2009 [citado 23 febrero 2020]. Disponible en: http://www.ilo.org/wcmsp5/groups/public/--americas/---ro-lima/---ilo-buenos_aires/documents/ publication/wcms_bai_pub_118.pdf

23. Ley de Seguridad y Salud en el Trabajo. Ley N 29783. El Peruano (19-8-2011). [citado 23 febrero 2020]. Disponible en: https://www.sunafil.gob.pe/ images/docs/normatividad/LEYDESEGURIDADSALUDTRABAJO-29783.pdf

24. Ley de Educación. Ley $N^{\circ} 28044$. El Peruano (287-2003). [citado 23 febrero 2020]. Disponible en: http://www.minedu.gob.pe/p/ley_general_de_educacion_28044.pdf

25. Artiles L, Otero J, Barrios I. Ciencia e investigación científica. En: Metodología de la investigación: Para ciencias de la Salud. La Habana, Cuba: Editorial de ciencias médicas; 2008.

26. Heyward, V. Diseño de programas para mejora la flexibilidad y cuidar la región lumbar. En: Evaluación de la aptitud física y prescripción de ejercicio. 5ta ed. Madrid, España: Editorial Médica Panamericana. 2008: 265-270.
27. López-Chicharro J. Fundamentos de fisiología del ejercicio. En: Fisiología clínica del Ejercicio. Madrid, España: Editorial Médica Panamericana. 2012: 14-15.

28. Carrasquero E. Adaptación y validación española del instrumento de percepción Cornell Musculoskeletal Discomfort Questionnaires (CMDQ). Desarrollo Gerencial. 2015;7(2):36-46. DOI: 10.17081/dege.7.2.1179

29. Erdinc O, Hot K, Ozkaya M. Turkish version of the Cornell Musculoskeletal Discomfort Questionnaire: Cross-cultural adaptation and validation. Work. 2011; 39:251-260. DOI: 10.3233/WOR-2011-1173

30. Ratzon N, Bar-Niv N, Froom P. The effect of a structured personalized ergonomic intervention program for hospital nurses with reported musculoskeletal pain: An assigned randomized control trial. Work. 2016; 54:367-377. DOI: 10.3233/WOR-162340

31. Karimi Z, Allahyari T, Azghani MR, Khalkhali $\mathrm{H}$. Influence of unstable footwear on lower leg muscle activity, volume change and subjective discomfort during prolonged standing. Appl Ergon. 2016; 53: 95-102. DOI: 10.1016/j.apergo.2015.09.003

32. Sezgin D, Esin M. Effects of a PRECEDE-PROCEED model based ergonomic risk management programme to reduce musculoskeletal symptoms of ICU nurses. Intensive Crit Care Nurs. 2018; 47:8997. DOI: 10.1016/j.iccn.2018.02.007 